\author{
L. A. Souza • F. F. Bellotti - T. Frederico - M. T. Yamashita • \\ Lauro Tomio
}

\title{
Scaling Limit Analysis of Borromean Halos
}

Received: 8 January 2016 / Accepted: 8 March 2016 / Published online: 13 April 2016

(C) Springer-Verlag Wien 2016

\begin{abstract}
The analysis of the core recoil momentum distribution of neutron-rich isotopes of light exotic nuclei is performed within a model of halo nuclei described by a core and two neutrons dominated by the $s$-wave channel. We adopt the renormalized three-body model with a zero-range force, which accounts for the Efimov physics. This model is applicable to nuclei with large two-neutron halos compared to the core size, and a neutron-core scattering length larger than the interaction range. The halo wave function in momentum space is obtained by using as inputs the two-neutron separation energy and the energies of the singlet neutron-neutron and neutron-core virtual states. Within our model, we obtain the momentum probability densities for the Borromean exotic nuclei Lithium-11 ( $\left.{ }^{11} \mathrm{Li}\right)$, Berylium-14 $\left({ }^{14} \mathrm{Be}\right)$ and Carbon-22 $\left({ }^{22} \mathrm{C}\right)$. A fair reproduction of the experimental data was obtained in the case of the core recoil momentum distribution of ${ }^{11} \mathrm{Li}$ and ${ }^{14} \mathrm{Be}$, without free parameters. By extending the model to ${ }^{22} \mathrm{C}$, the combined analysis of the core momentum distribution and matter radius suggest (i) a ${ }^{21} \mathrm{C}$ virtual state well below $1 \mathrm{MeV}$; (ii) an overestimation of the extracted matter ${ }^{22} \mathrm{C}$ radius; and (iii) a two-neutron separation energy between 100 and $400 \mathrm{keV}$.
\end{abstract}

\section{Introduction}

The large reaction cross section of Carbon-22 $\left({ }^{22} \mathrm{C}\right)$ observed by Tanaka et al. [1], in the collision of ${ }^{22} \mathrm{C}$ on a liquid hydrogen target at $40 \mathrm{~A} \mathrm{MeV}$ compared to the neighbour carbon nuclides, ${ }^{19} \mathrm{C}$ and ${ }^{20} \mathrm{C}$, triggered a

\author{
L. A. Souza $\cdot$ T. Frederico $(\varangle) \cdot$ L. Tomio \\ Instituto Tecnológico de Aeronáutica, DCTA, São José dos Campos 12228-900, Brazil \\ E-mail: tobias@ita.br \\ L. A. Souza \\ E-mail: lucasufsj@gmail.com \\ L. Tomio \\ E-mail: tomio@ift.unesp.br \\ F. F. Bellotti \\ Department of Physics and Astronomy, Aarhus University, 8000 Aarhus C, Denmark \\ F. F. Bellotti \\ Instituto de Fomento e Coordenação Industrial, São José dos Campos, SP 12228-901, Brazil \\ E-mail: ffbellotti@gmail.com
}

M. T. Yamashita $\cdot$ L. Tomio

Instituto de Física Teórica, UNESP, São Paulo 01156-970, Brazil

E-mail: yamashita@ift.unesp.br 
theoretical discussion [2-6] about the possible constraint that the associated extracted matter root-mean-square (rms) radius $\sqrt{\left\langle r_{m}^{2}\right\rangle}$ can have on the two-neutron separation energy, $S_{2 n}$. From the experimental point of view the two-neutron separation energy of ${ }^{22} \mathrm{C}$ is not well known. A recent atomic mass evaluation (Ame2012), reported in Ref. [7], gives $S_{2 n}=110$ (60) $\mathrm{keV}$ for ${ }^{22} \mathrm{C}$. And, from a mass measurement [8], $S_{2 n}=-0.14$ (46) $\mathrm{keV}$. There is also an indirect evidence that ${ }^{22} \mathrm{C}$ could be bound by less than $70 \mathrm{keV}$ [9].

Such reaction cross-section, analysed with a finite-range Glauber calculation under an optical-limit approximation, suggested that $\sqrt{\left\langle r_{m}^{2}\right\rangle}=5.4 \pm 0.9 \mathrm{fm}$ [1]. Their analysis showed that the two-valence neutrons prefer to occupy the $s_{1 / 2}$ orbital. Considering the observed ${ }^{22} \mathrm{C}$ matter radius together with the ${ }^{20} \mathrm{C}$ one, the following estimate was given in Ref. [2] for the radius of the neutron halo orbit:

$$
\sqrt{\left\langle r_{n}^{2}\right\rangle}=\sqrt{\frac{22}{2}} \sqrt{\left\langle r_{m}^{2}\left[{ }^{22} C\right]\right\rangle-\frac{20}{22}\left\langle r_{m}^{2}\left[{ }^{20} C\right]\right\rangle}=15 \pm 4 \mathrm{fm}
$$

where $\sqrt{\left\langle r_{m}^{2}\left[{ }^{20} C\right]\right\rangle}=2.98(5) \mathrm{fm}[10]$. The ${ }^{22} \mathrm{C}$ rms matter radius is used as a constraint to the poorly known experimental value of the two-neutron separation energy, in a renormalized zero-range three-body model [2], in a shell model approach [3], in a three-body cluster model [4], and within an effective field theory with contact interactions [5]. All these approaches agree to a value around or below $100 \mathrm{keV}$. However, independent information is relevant to cross check such estimations. Indeed, in Ref. [4], a strong correlation between the soft dipole mode in the Coulomb fragmentation process of ${ }^{22} \mathrm{C}$ and $S_{2 n}$ was also shown.

Comparison between experimental data for the core recoil momentum distributions of ${ }^{22} \mathrm{C}[11]$ and Lithium$11\left({ }^{11} \mathrm{Li}\right)[12]$ indicates similar sizes for their two-neutron halos, which suggests that the matter radius of the carbon isotope could be overestimated (see Ref. [13], for further discussion). This motivates us to study the properties of low-momentum distributions in two-neutron halo light exotic nucleus, which are dominated by $s$-wave short-range two-body interactions in the neutron-neutron $(n-n)$ and neutron-core $(n-c)$ subsystems.

In the present work, the halo properties are analysed by using correlations between observables or scaling functions, involving the width of the core recoil distribution, $S_{2 n}$, the energies of the $s$-wave virtual states of the singlet spin $n-n$ system and ${ }^{21} \mathrm{C}$. Furthermore, correlations between the matter radius and the quantities mentioned before are also used in our combined analysis of the existing data for the width, matter radius and the virtual state of ${ }^{21} \mathrm{C}$. Our interest is to constrain the parameters associated with the halo structure and two-neutron separation energy based on these data fitted to three-body model calculations, by adding to the work presented in Ref. [14] other universal correlations of the width of the core momentum distribution and ${ }^{22} \mathrm{C}$ matter radius.

The model calculations for the core recoil momentum distribution are performed within a zero-range threebody model applied to a neutron-neutron-core $(n-n-c)$ system, that fully accounts for the so-called Efimov physics [15-17]. The detailed formulation of the momentum distributions was given in [18] and the inputs of the renormalized zero-range model are the scattering lengths, and one three-body scale, which is given by the two-neutron separation energy. Therefore, three low-energy observables are enough to determine another low-energy quantity through an appropriate universal scaling function computed within the zero-range model. Following that, we established the above mentioned correlations between two $s$-wave halo observable, like, e.g., the width of the momentum distribution with $S_{2 n}$, matter radius with $S_{2 n}$, and the width and matter radius. In particular, this last one was useful to constrain the virtual state energy of ${ }^{21} \mathrm{C}$, as will be discussed in detail.

In our work, the data for the core recoil momentum distribution extracted from the halo fragmentation reaction are interpreted on the basis of the Serber model [19], where the peak around zero momentum is proportional to the momentum distribution. We test the reliability of our analysis with ${ }^{11} \mathrm{Li}$ (see e.g. [12]) and ${ }^{14} \mathrm{Be}$, as the low energy parameters used as input to our calculations are reasonably known. The recent experimental results for ${ }^{20} \mathrm{C}$ and ${ }^{22} \mathrm{C}$ [11] allow us, in principle, to constrain $S_{2 n}$ and the matter radius of ${ }^{22} \mathrm{C}$. In addition, we consider the constraint to the virtual state of ${ }^{21} \mathrm{C}$ by also computing the matter radius, which has a given value in Ref. [1]. An issue beyond the present work is the consideration of the range corrections to the scaling functions, either by calculations with finite range potentials or along the lines suggested in Ref. [20], as well as in a series of recent works [21-24], which we leave for a future investigation.

The paper is organized as follows. In Sect. 2 , the experimental ${ }^{22} \mathrm{C}$ core recoil momentum distribution is revised and the width associated with the two-neutron halo is singled out. In Sect. 3, the scaling relations for the $n-n-c$ system close to the unitary limit is presented. In Sect. 4, the scaling analysis for the ${ }^{22} \mathrm{C}$ halo is performed in light of the experimental result for the momentum width. Finally, in Sect. 5, we have our summary. 


\section{Core Recoil Momentum Distribution in ${ }^{22} \mathrm{C}$ : Phenomenological Analysis}

The information on the core recoil momentum distribution in two-neutron halo nuclei is assessed through the removal cross-section from the collision of these radioactive exotic nuclei with nuclear targets at few hundred $\mathrm{MeV} /$ nucleon (see e.g. [25]). For neutrons flying out from $s$-states, these removal cross-sections shows a characteristic narrow peak at zero core recoil momentum, and a width that features the weak binding of the neutrons to the core. In the example of ${ }^{11} \mathrm{Li}$ [12], it is visible the contribution of distributions with a narrow and wide widths. The narrow momentum distribution is associated with the two neutrons in the halo state, and the wide one to the neutrons in the inner orbits in ${ }^{11} \mathrm{Li}$ and inside the ${ }^{9} \mathrm{Li}$ core. Adopting such interpretation, the final core momentum distribution is an incoherent sum of two distributions one narrow and another wide. Of course this picture is based on a large separation between the halo neutron orbit and the inner ones, implying in a small overlap among them, and suppression of the coherent terms in the removal cross-section. By scaling arguments, the width of the narrow peak in ${ }^{11} \mathrm{Li}$ should be of the order of $\sqrt{m_{n} S_{2 n}}=18 \mathrm{MeV} / \mathrm{c}$, where $m_{n}$ is the nucleon mass. This is quite close to the experimental fit, 21(3) MeV/c, given in Ref. [12]. For such estimate we use the two-neutron separation energy of $S_{2 n}=369.15(65) \mathrm{keV}$ [26].

The above description applied to ${ }^{22} \mathrm{C}$ as formed by two neutrons in a weakly-bound halo with a core of ${ }^{20} \mathrm{C}$, one expect that the core recoil momentum distribution presents itself as a narrow peak on the top of a wide one, once the halo neutrons occupy an $s$ state. The narrow and wide peaks should be associated with well separated length or momentum scales, in order to characterize a true two-neutron halo state. We can resort to the recent experimental information on two neutron removal cross-section [11] of ${ }^{22} \mathrm{C}$ and ${ }^{20} \mathrm{C}$ colliding with a carbon target at $240 \mathrm{MeV} / \mathrm{A}$. The experimental resolution for both cases are very close. This allows us to make a comparison among the corresponding experimental removal cross-sections, which are shown in the left panel of Fig. 1 as functions of the inclusive parallel momentum, $P_{\|}$, of the respective core (e.g., ${ }^{20} \mathrm{C}$ in case of ${ }^{22} \mathrm{C}$; and ${ }^{18} \mathrm{C}$ in case of ${ }^{20} \mathrm{C}$ ). Apart from small kinematical corrections, it is quite clear that the wide distribution corresponds to neutrons removed from the core of ${ }^{22} \mathrm{C}$, with a narrow peak being clearly characterized.

To express such separation among the length/momentum scales of the halo state and the inner core orbits in ${ }^{22} \mathrm{C}$, we introduce a phenomenological quantity, which gives the difference the two-neutron removal crosssections in ${ }^{22} \mathrm{C}$ and ${ }^{20} \mathrm{C}$

$$
\Delta\left(P_{\|}^{2}\right)=\frac{d \sigma}{d P_{\|}}\left[{ }^{22} C\right]-\frac{d \sigma}{d P_{\|}}\left[{ }^{20} C\right],
$$

which should be associated with the two-neutron halo property of ${ }^{22} \mathrm{C}$, as the reaction rate associated with the removal of neutrons from the inner orbits is expected to be the same as in ${ }^{20} \mathrm{C}$. The core recoil momentum distribution is parametrized as a normal distribution

$$
\Delta\left(p^{2}\right)=N \exp \left(-\frac{p^{2}}{2 s^{2}}\right)
$$
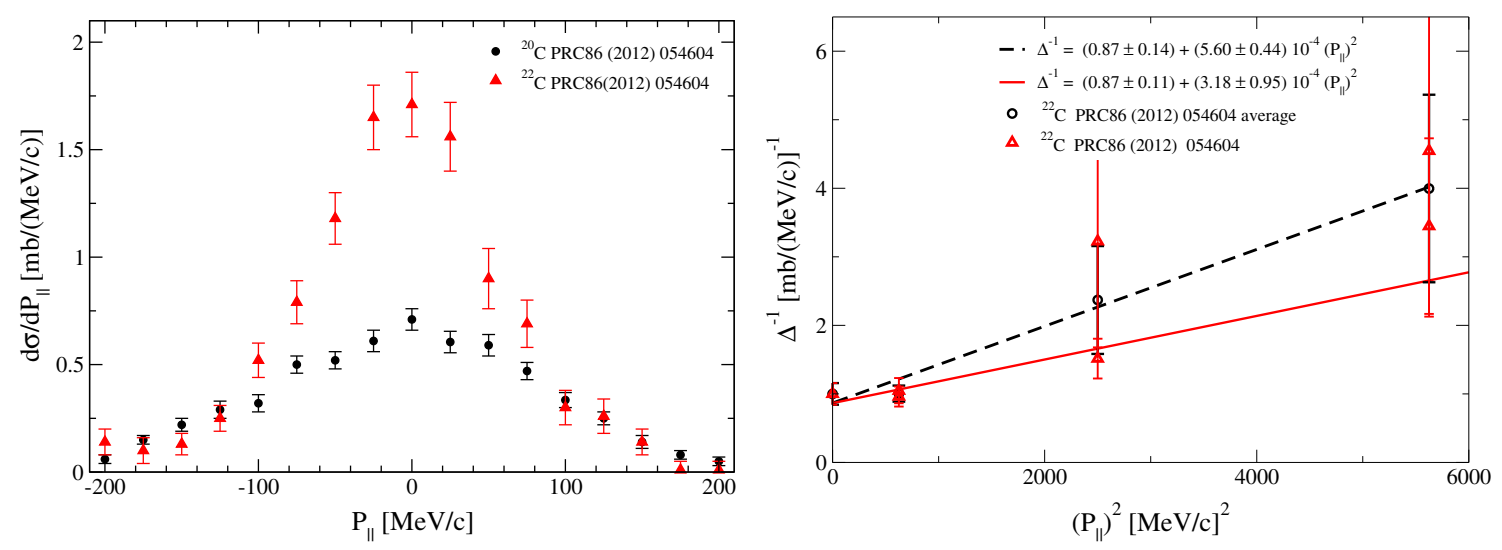

Fig. 1 (color online) Left-frame Experimental data for two-neutron removal cross-section from ${ }^{22} \mathrm{C}$ (red-triangles) and from ${ }^{20} \mathrm{C}$ (black-circles), obtained in a collision with a carbon target at $240 \mathrm{MeV} / \mathrm{A}$, given as a function of the rest frame core recoil momentum [11]. Right-frame The inverse of the difference between the removal cross-sections of ${ }^{22} \mathrm{C}$ and ${ }^{20} \mathrm{C}$, given by Eqs. (4) (black-dashed line) and (5) (solid-red line) are shown as functions of the squared recoil core momentum. Circles (triangles) represent the averaged difference for $P_{\|}>0$ and $P_{\|}<0$, fitted by the dashed (solid) straight line 
where the variance is given by $\mathrm{s}^{2}$, with the corresponding standard deviation $\mathrm{s}=\sqrt{\mathrm{s}^{2}}$ associated with the Full Width at Half Maximum (FWHM) distribution, $w \equiv \sqrt{8 \ln 2}$ s. $N$ is a normalization from the data. In the right frame of Fig. 1, we plot the data for $\Delta^{-1}$ as a function of $P_{\|}^{2}$. We also present the averaged data for positive and negative $P_{\|}$, shown by empty circles. A straight line fit is suggested by data. For $p$ given in units of $\mathrm{MeV} / \mathrm{c}$, with $p \equiv(\bar{p}) \mathrm{MeV} / \mathrm{c}$, considering the averaged experimental values, we obtain

$$
\Delta^{-1}\left(p^{2}\right)=\left\{(0.87 \pm 0.14)+(5.60 \pm 0.44) 10^{-4} \bar{p}^{2}\right\}[\mathrm{mb} /(\mathrm{MeV} / \mathrm{c})]^{-1} .
$$

The result for the FWHM is $w=79 \pm 22 \mathrm{MeV} / \mathrm{c}$, with the associated $\mathrm{s}_{a v}=34 \pm 9 \mathrm{MeV} / \mathrm{c}$. Taking into account the experimental resolution of $\mathrm{s}_{\exp }=27 \mathrm{MeV} / \mathrm{c}$ [11], we obtain $\mathrm{s}_{a v}=20 \pm 15 \mathrm{MeV} / \mathrm{c}$. The fitting of the difference without the average is given by

$$
\Delta^{-1}\left(p^{2}\right)=\left\{(0.87 \pm 0.11)+(3.18 \pm 0.95) 10^{-4} \bar{p}^{2}\right\}[\mathrm{mb} /(\mathrm{MeV} / \mathrm{c})]^{-1},
$$

such that for the FWHM we have $w=104 \pm 39 \mathrm{MeV} / \mathrm{c}$, with the corresponding $\mathrm{s}=44 \pm 16 \mathrm{MeV} / \mathrm{c}$. After considering the experimental resolution this value is reduced to $\mathrm{s}=34 \pm 21 \mathrm{MeV} / \mathrm{c}$. The FWHM quoted in [11] for the narrow peak of ${ }^{22} \mathrm{C}$ is $73 \mathrm{MeV} / \mathrm{c}$, with $\mathrm{s}=31 \mathrm{MeV} / \mathrm{c}$, compatible with our phenomenological analysis.

Naively, one could estimate that the narrow peak leads to $S_{2 n} \sim \mathrm{s}^{2} / m_{n}$. In this case, for $\mathrm{s}=(20,34,31)$ $\mathrm{MeV} / \mathrm{c}$ the corresponding values of $S_{2 n}$ are given by $(0.4,1.2,1) \mathrm{MeV}$. These values appear to be somewhat large according to the analysis performed in [2-5], where the suggested upper limit for $S_{2 n}$ is $\sim 0.1 \mathrm{MeV}$ to achieve consistency with the extracted rms matter radius of $5.4 \pm 9 \mathrm{fm}$ [1]. In order to refine our estimate of $S_{2 n}$, we will rely on three-body calculations in the zero-range limit, where the universal scaling laws are defined and computed, and in addition the errors from our fitting procedure have to be considered.

\section{The Neutron-Neutron-Core System Close to the Unitary Limit}

Our goal here is to present an analysis of the core recoil momentum distributions of light two-neutron halonuclei based on scaling laws computed for large scattering lengths, close to the unitary limit and dominated by the so-called Efimov physics [15-17]. We adopt a renormalized three-body zero-range model, with the halo nucleus described as two neutrons with an inert core $(n-n-c)$, which is appropriate for the analysis of such weakly-bound systems, where the $n-n$ and the $n-c$ interactions are dominated by $s$-wave states (see e.g. [27-29]). Examples of two-neutron halo nuclei, which follow these assumptions, are ${ }^{11} \mathrm{Li},{ }^{14} \mathrm{Be}$ and the carbon systems ${ }^{20} \mathrm{C}$ and ${ }^{22} \mathrm{C}$. We study the width of the core recoil momentum distribution and make use of the existing data for ${ }^{11} \mathrm{Li}[12]$ and ${ }^{14} \mathrm{Be}$ [30] to validate the model, and then analyse the case of ${ }^{22} \mathrm{C}$ [11]. The momentum distribution is extracted from the halo fragmentation on nuclear targets (see e.g. Ref. [25]). The present analysis details our recent study [14] and emphasizes the ${ }^{22} \mathrm{C}$ case.

The core recoil momentum distribution in the $n-n-c$ system is given by:

$$
n\left(q_{c}\right)=\int d^{3} p_{c}\left|\left\langle\mathbf{q}_{c} \mathbf{p}_{c} \mid \Psi\right\rangle\right|^{2},
$$

where the relative momentum of the core to the $n-n$ subsystem is $\mathbf{q}_{c}$ and the relative momentum between the neutrons is $\mathbf{p}_{c}$, which is integrated above. Detailed expressions are given in [18], within the renormalized three-body zero-range model. It requires as inputs the scattering lengths for the $n-c$ and $n-n$ systems, besides one three-body $(n-n-c)$ scale, which will be chosen as $S_{2 n}$. In the unitary limit, besides the core mass $A$, the only scale that remains is the three-body binging energy. The geometrically separated tower of Efimov states is completely determined by such energy, as a necessary requirement from the relation between the Efimov effect and the Thomas collapse [31]. In what follows, instead of the scattering lengths, we are going to refer to the corresponding two-body virtual state energies (as the present study is limited to Borromean $n-n-c$ systems), which are taken by their absolute (positive) quantites $E_{n c}$ and $E_{n n}$. The $n-n$ virtual state energy in $s$-wave is known and in general will be fixed to $143 \mathrm{keV}$.

The momentum space $s$-wave three-body wave function $(|\Psi\rangle)$, for the renormalized zero-range three-body model applied to the two-neutron halo nuclei, can be written in terms of two spectator functions, $\chi_{n n}\left(q_{c}\right)$ and $\chi_{n c}\left(\left|\mathbf{p}_{c} \pm \frac{\mathbf{q}_{c}}{2}\right|\right)$ [28], where the bound-state three-body energy is replaced by the two-neutron separation energy $S_{2 n}$, such that

$$
\left\langle\mathbf{q}_{c} \mathbf{p}_{c} \mid \Psi\right\rangle=\frac{\chi_{n n}\left(q_{c}\right)+\chi_{n c}\left(\left|\mathbf{p}_{c}-\frac{\mathbf{q}_{c}}{2}\right|\right)+\chi_{n c}\left(\left|\mathbf{p}_{c}+\frac{\mathbf{q}_{c}}{2}\right|\right)}{S_{2 n}+H_{0}} .
$$


In the above, the corresponding free Hamiltonian is given by

$$
H_{0} \equiv \frac{p_{c}^{2}}{2 m_{n n}}+\frac{q_{c}^{2}}{2 m_{n n c}},
$$

where $m_{n n}$ and $m_{n n c}$ are the reduced masses, given by $m_{n n}=m_{n} / 2$ and $m_{n n c}=2 A m_{n} /(A+2)$, with $A \equiv m_{c} / m_{n}$. The core is assumed structureless with the two neutrons in a singlet spin state to ensure the antisymmetrization of the halo wave function.

The spectator functions in the renormalized three-body model are the solution of a coupled set of homogeneous integral equations, which correspond to the Faddeev equations for the zero-range interaction subtracted at a given scale. Besides the $n-n$ and $n-c$ energies, within the zero-range model we need to provide a regularization scale, which is given by an energy parameter $-\mu^{2}$. This regularization is done by fixing $S_{2 n}$. The value of $\mu$ can be moved towards infinity, generating a tower of Thomas-Efimov excited states. The log-periodic behaviour of the spectator functions appear in the ultraviolet region, not affecting the low momentum part of the wave function that indeed runs to a limit cycle. This behaviour is explored in detail in [18]. A universal ratio between the magnitude of the log-periodic spectator functions in the limit of $\mu \rightarrow \infty$, which is a function of only the mass ratio $A$, was also found. We noticed that in practice the first cycle is enough to get the properties of the wave function in the infrared momentum region, which is acceptable for our purposes of computing the width of the momentum distribution. This happens because in the actual cases of for example, ${ }^{11} \mathrm{Li}$ and ${ }^{22} \mathrm{C}$, one finds that $\mu^{2} \gg S_{2 n}$. Therefore, our calculation of the scaling law for the width of the core recoil momentum distribution is close to the universal limit cycle already in the first cycle. We have checked in [14] that correlations between the width and $S_{2 n}$ of the ground, first and second excited states are quite close and in practice the limit cycle for this scaling law is achieved with the ground state calculation, of the renormalized zero-range three-body model (see e.g. [28]), namely given by the subtracted Skorniakov and Ter-Martirosian equations for mass imbalanced systems [32].

The utility of a scaling function in few-body physics was recognized long ago by Phillips [33] in the analysis of the doublet neutron-deuteron scattering length through the correlation with the triton binding energy, which he found quite model independent. In the 80's Efimov showed that the origin of the model independence of the "Phillips line" is the universal long range potential, which emerges close to the unitary limit (infinite two-body scattering length). Our approach just follows that idea, searching for the correlations between any other two three-body low-energy $s$-wave observable, by using the zero-range model to compute the associated scaling law. One can use a scaling function to estimate for example the value of $S_{2 n}$ or s, knowing one of them and eventually use it to constrain some other poorly known low-energy observable, such as a subsystem scattering length.

The scaling function for the width of the core recoil momentum distribution, gives a correlation between $\mathrm{s}$ and the energies $S_{2 n}, E_{n c}$ and $E_{n n}$, which can be written in a general form as

$$
\mathrm{s}=\sqrt{S_{2 n} m_{n}} \mathcal{S}_{c}\left( \pm \sqrt{\frac{E_{n n}}{S_{2 n}}}, \pm \sqrt{\frac{E_{n c}}{S_{2 n}}} ; A\right),
$$

where the + and - signs refer to the bound and virtual subsystem energies, respectively. In the present specific Borromean three-body case, we have only virtual two-body subsystems, such that both signs are negative in Eq. (9), with $E_{n n}$ fixed to the $n-n$ virtual state. For the momentum distribution width, the scaling function (9) is the limit cycle of the correlation function associated with s as a function of $E_{n n}, E_{n c}$ and $S_{2 n}$, when the three-body ultraviolet (UV) cut-off is driven to infinite in the three-body integral equations, or equally the subsystem energies are let to zero with a fixed UV cut-off. Similar procedure is performed within a renormalized zero-range three-body model, in the subtracted integral equations, where the subtraction energy is fixed and the two-body scattering lengths are driven towards infinite. In practice, both procedures provides very close results, as shown in Ref. [34]. In the exact Efimov limit $\left(E_{n n}=E_{n c}=0\right)$, the width is a universal function of the mass number $A$,

$$
\mathrm{s}=\sqrt{S_{2 n} m_{n}} \mathcal{S}_{c}(0,0, A) \approx 1.154 \sqrt{S_{2 n} m_{n}} \frac{\sqrt{A} / \pi+A}{1+\sqrt{A} / \pi+A} .
$$

The scaling function given by (9) is associated to the limit cycle for the correlation between s and $S_{2 n}$, which in principle can be computed for an Efimov state with large excitation. The approximate expression (10) was obtained in [14], within a fit procedure. 
Table 1 Theoretical values of $\mathrm{s}_{t h}$, obtained from the scaling function (10), are compared with the corresponding experimental values, $\mathrm{s}_{\text {exp }}$, which were extracted from the core recoil momentum distribution of the Borromean halo nuclei ${ }^{11} \mathrm{Li},{ }^{14} \mathrm{Be}$ and ${ }^{22} \mathrm{C}$

\begin{tabular}{llll}
\hline Borromean nuclei & $S_{2 n}[\mathrm{keV}][7]$ & $\mathrm{s}_{t h}[\mathrm{MeV} / \mathrm{c}]$ & $\mathrm{s}_{\exp }[\mathrm{MeV} / \mathrm{c}]$ \\
\hline${ }^{11} \mathrm{Li}$ & $369.28 \pm 0.64$ & 19.5 & $21 \pm 3[12]$ \\
${ }^{14} \mathrm{Be}$ & $1265.89 \pm 132.26$ & $37 \pm 2$ & $39.3 \pm 1.1[30]$ \\
${ }^{22} \mathrm{C}$ & $110 \pm 60$ & $11 \pm 3$ & $31[11] ; 20 \pm 15(4) ; 34 \pm 21(5)$ \\
\hline
\end{tabular}

In the case of ${ }^{22} \mathrm{C}$, we consider the averaged expression (4), as well as (5) without averaging

In Table 1 we compare the results of Eq. (10) for $\mathrm{s}$ in the case of the Borromean halos ${ }^{11} \mathrm{Li},{ }^{14} \mathrm{Be}$ and ${ }^{22} \mathrm{C}$. The comparison is illustrative as we are considering only the unitary limit, and therefore disregarding the small virtual state energies of the subsystems. The dependence of $s$ on the subsystems energies is weak, and small corrections are present, as we have verified in [14]. In the case of ${ }^{22} \mathrm{C}$ the calculation is poor compared to the experimental value of $\mathrm{s}$ from [11], while our phenomenological analysis, with values of s obtained from the fits (4) and (5), is able to overlap with the theoretical estimation due to the large uncertainties. We will present more results in the next section and return to this discussion, despite the small effects on s from $E_{n n}$ and $E_{n c}$ for a given two neutron separation energy. We point out that the model is validated by the comparison with experimental values in the cases of ${ }^{11} \mathrm{Li}$ and ${ }^{14} \mathrm{Be}$.

\section{Scaling Analysis of ${ }^{22} \mathrm{C}$ Halo}

We apply the $n-n-c$ model to describe the halo of ${ }^{22} \mathrm{C}$ and the results for the core recoil momentum distribution s expressed by the scaling function (9) are shown in the left-frame of Fig. 2. We have kept fixed $E_{n n}=143$ $\mathrm{keV}$ and varied the energy of the virtual $s$-state of ${ }^{21} \mathrm{C}$ by considering $E_{n c} / E_{n n}$ equal to $0,2,6$ and 10 . We allow a variation of $E_{n c}$, by including the region where it is $1 \mathrm{MeV}$, as indicated in Ref. [9], to spam different possibilities. The variation of $E_{n c}$ with fixed $S_{2 n}$ and $E_{n n}$ does not show a dramatic effect, as we have pointed out in [14]. We also present the scaling function at the unitary limit (10), and is quite close to the results with finite values of $E_{n c}$ and $E_{n n}$, showing very clearly in the model that $S_{2 n}$ is the dominating scale in the core recoil momentum distribution in the three-body halo at low momentum. In addition, for large values of $S_{2 n}$, i.e., when $S_{2 n}$ is much larger than $E_{n n}$ and $E_{n c}$, the scaling function tends to approach the unitary result, as expected.

The matter radius is another quantity that we can compute from the halo neutron orbit radius with respect to the center of mass, $\sqrt{\left\langle r_{n}^{2}\right\rangle}$, which can also be written in terms of a scaling function [35], such that
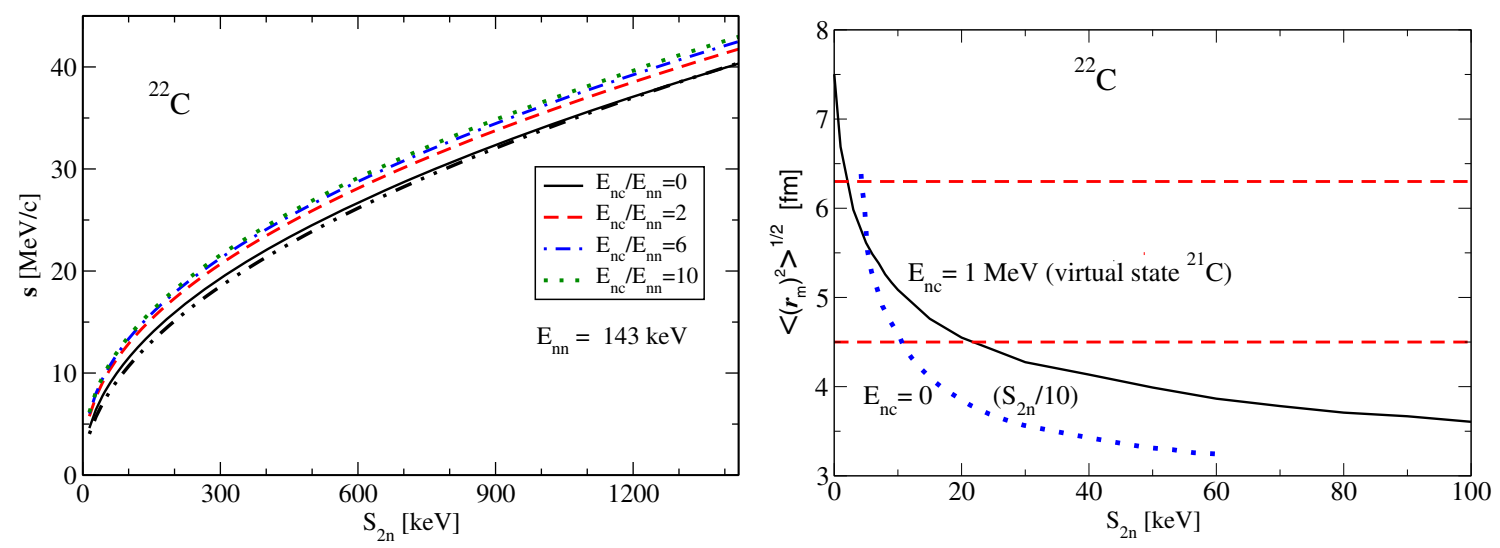

Fig. 2 (color online) Left-frame Scaling plot for the width s as a function of $S_{2 n}$, for ${ }^{22} \mathrm{C}$ with different ratios $E_{n c} / E_{n n}$. The results from the scaling law given by Eq. (10) are shown by the dot-dot-dashed line (lower one). Right-frame Root mean square matter radius for ${ }^{22} \mathrm{C}$ as a function of $S_{2 n}$ for the $n-c$ virtual state energy fixed at $1 \mathrm{MeV}$ (solid line) and 0 (dotted line). The $n-n$ virtual state energy is fixed to $143 \mathrm{keV}$. The horizontal dashed lines represents the value of $\sqrt{\left\langle r_{m}^{2}\right\rangle}=5.4 \pm 0.9 \mathrm{fm}$ extracted in the experiment of Ref. [1] 

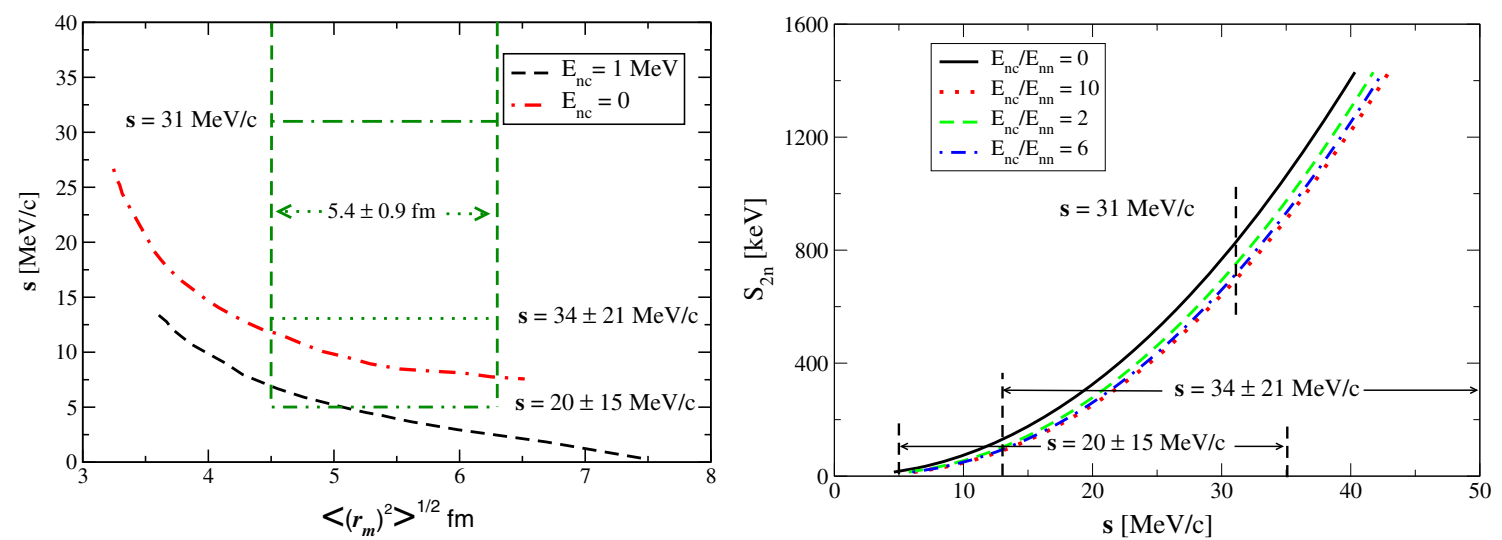

Fig. 3 (color online) Left-frame Scaling plot for the width s of the variance of the core recoil momentum distribution in ${ }^{22} \mathrm{C}$ as a function function of the root mean square matter radius, $\sqrt{\left\langle r_{m}^{2}\right\rangle}$, for $E_{n c}=0$ (dot-dashed line) and for $E_{n c}=1 \mathrm{MeV}$ (dashed line), with a fixed $n-n$ virtual state energy of $143 \mathrm{keV}$. The extracted matter radius, $\sqrt{\left\langle r_{m}^{2}\right\rangle}=5.4 \pm 0.9 \mathrm{fm}$, from Ref. [1] is indicated in the figure. The values of s shown in the figure: $31 \mathrm{MeV} / \mathrm{c}$ [11] (horizontal dot-dashed line), lower bound for $\mathrm{s}_{a v}=20 \pm 15$ $\mathrm{MeV} / \mathrm{c}$ (double-dot-dashed line) derived from Eq. (4) and lower bound for $\mathrm{s}=34 \pm 21 \mathrm{MeV} / \mathrm{c}$ (dotted line) obtained from Eq. (5). Right-frame Two-neutron separation energy $S_{2 n}$ in ${ }^{22} \mathrm{C}$ as a function of s for fixed $n-n$ virtual state energy of $143 \mathrm{keV}$ and different values of the virtual $n-c$ energy. Calculations for $E_{n c} / E_{n n}$ values of 0 (solid line), 2 (dashed line), 6 (dot-dashed line) and 10 (dotted line)

$$
\sqrt{\left\langle r_{n}^{2}\right\rangle}=\sqrt{\frac{m_{n}}{\hbar^{2}} S_{2 n}} \mathcal{R}_{n}\left( \pm \sqrt{\frac{E_{n n}}{S_{2 n}}}, \pm \sqrt{\frac{E_{n c}}{S_{2 n}}} ; A\right)
$$

This allows us to compute the matter radius, as

$$
\sqrt{\left\langle r_{m}^{2}\left[{ }^{22} C\right]\right\rangle}=\sqrt{\frac{2}{22}\left\langle r_{n}^{2}\right\rangle+\frac{20}{22}\left\langle r_{m}^{2}\left[{ }^{20} C\right]\right\rangle} .
$$

Only by considering the ${ }^{22} \mathrm{C}$ extracted matter radius of 5.4(9) fm [1], the conclusion of [2], based on the model we are following here, was that $S_{2 n}$ is below $100 \mathrm{keV}$. However, in view of the new data on the core momentum distribution, we ought to include it on the analysis of the two-neutron separation energy. The right frame of Fig. 2 summarize the findings of [2], where we plot the correlations between $\sqrt{\left\langle r_{m}^{2}\right\rangle}$ and $S_{2 n}$. The virtual $s$-state energy of ${ }^{21} \mathrm{C}$ is chosen to be 0 and $1 \mathrm{MeV}$ [9], with fixed $E_{n n}=143 \mathrm{keV}$. In the case of $E_{n c}=0$, the matter radius is compatible with $S_{2 n}<100 \mathrm{keV}$, while by increasing the $E_{n c}$ the upper limit for $S_{2 n}$ decreases, as shown by the results obtained for $E_{n c}=1 \mathrm{MeV}$. This happens because the interaction becomes weaker as the $E_{n c}$ increases; and, for a given three-body binding energy, the system has to shrink [35]. This is the effect behind the decrease of the possible values of $S_{2 n}$, when the ${ }^{21} \mathrm{C}$ virtual state energy is varied from 0 to $1 \mathrm{MeV}$.

We have data extracted from experiments for the width s of the core recoil momentum distribution and the matter radius of ${ }^{22} \mathrm{C}$. Then, we just built the correlation between these quantities and analyse the data in view of the scaling function of our model. The left-frame of Fig. 3 shows the correlation between the rms matter radius and $\mathrm{s}$ for the values of $E_{n c}$ equal to 0 and $1 \mathrm{MeV}$. For a given matter radius s increase by changing $E_{n c}$ from 0 of to $1 \mathrm{MeV}$. The variation is noticeable, about a factor of 2 . That effect is a reminiscent of the contraction of the wave function by decreasing the energy $E_{n c}$. The region delimited by the matter radius of $5.4 \pm .9 \mathrm{fm}$ and the three values for s, namely, $31 \mathrm{MeV} / \mathrm{c}[11], 20 \pm 15 \mathrm{MeV} / \mathrm{c}$ from the fit (4) and $34 \pm 21$ $\mathrm{MeV} / \mathrm{c}$ from the fit (5) is shown in the left-frame of the figure. It looks that the value of $E_{n c}=1 \mathrm{MeV}$ is too large from the consistency between the scaling function, the matter radius and the lower bound of $\mathrm{s}=20 \pm 15$ $\mathrm{MeV} / \mathrm{c}$. Just by adopting $\mathrm{s}=31 \mathrm{MeV} / \mathrm{c}$ the matter radius basically goes to the core radius $\sim 3 \mathrm{fm}$. The lower bounds of $20 \pm 15$ and $34 \pm 21 \mathrm{MeV} / \mathrm{c}$, taken together with the scaling function seems consistent with the matter radius. However, the central values of s suggests that the matter radius is overestimated. In order to be consistent with the matter radius the distribution should be quite narrow according to the scaling function.

The right-frame of Fig. 3 completes our analysis of the two-neutron separation energy in ${ }^{22} \mathrm{C}$. We show $S_{2 n}$ as a function of s. The data values for s suggest that $S_{2 n}$ could have larger values than estimated before, and stay above $100 \mathrm{keV}$. Taking as reference the data with the average between positive and negative momentum 
around the maximum of core momentum distribution peak, i.e., $\mathrm{s}=20 \pm 15 \mathrm{MeV} / \mathrm{c}$, one gets $S_{2 n} \sim 400 \mathrm{keV}$ and $\sqrt{\left\langle r_{m}^{2}\right\rangle} \sim 3.5 \mathrm{fm}$. The values of s equal to $31 \mathrm{MeV} / \mathrm{c}$ [11] and 34 $\pm 21 \mathrm{MeV} / \mathrm{c}$ (5) suggest much larger values of the two-neutron separation energy about 800 and $900 \mathrm{keV}$, respectively. The neutron halo of ${ }^{22} \mathrm{C}$ in this case would be quite small compared to the corresponding one in ${ }^{11} \mathrm{Li}$, and possibly would not justify the increase of the reaction cross section of ${ }^{22} \mathrm{C}$ compared to the neighbour nuclei, ${ }^{19} \mathrm{C}$ and ${ }^{20} \mathrm{C}$, as observed by Tanaka and collaborators [1], in the collision of ${ }^{22} \mathrm{C}$ on a liquid hydrogen target. Our analysis, considering this fact together with the clear presence of the narrow peak in the core recoil momentum distribution, leads to $\mathrm{s}<20$ $\mathrm{Mev} / \mathrm{c}, S_{2 n}<400 \mathrm{keV}$ and $\sqrt{\left\langle r_{m}^{2}\right\rangle}>3.5 \mathrm{fm}$. The sensitivity to the virtual $s$-state energy of ${ }^{21} \mathrm{C}$ is quite small in s for a given $S_{2 n}$, but it has a noticeable effect in the matter radius of ${ }^{22} \mathrm{C}$ shrinking considerably the halo as $E_{n c}$ runs from 0 to $1 \mathrm{MeV}$. That tension between the halo size and the virtual state of ${ }^{21} \mathrm{C}$ indicates that smaller values of $E_{n c}$ below $1 \mathrm{MeV}$ are preferred by our analysis. We rely on the dominance of the Efimov physics on the halo properties and in the Serber model to interpret the data on the core recoil momentum distribution.

\section{Conclusions}

The core recoil momentum distribution of Borromean neutron-rich isotopes of light exotic nuclei is studied within a three-body model constituted by a core and two neutrons, where the interaction is dominated by the $s$-wave channel. We adopt a renormalized three-body model with a zero-range force, which naturally accounts for the universality of the Efimov physics. Our model is adequate to describe a halo nucleus with two neutrons weakly bound to a core, where the halo neutrons explore with high probability the classically forbidden region well outside the core. In addition, the $n-c$ scattering length should be larger than the interaction range. These conditions are well satisfied by the Borromean halo of ${ }^{11} \mathrm{Li}$, the prototype of a weakly-bound radioactive halo nucleus at the neutron drip line.

The core momentum distribution for the halo wave function is obtained from the numerical solution of the subtracted homogeneous Skorniakov and Ter-Martorisyan coupled equations for the zero-range interaction in the the mass imbalanced case. The inputs are the two-neutron separation energy and the energies of the singlet $n-n$ and $n-c$ virtual states. We calculated the momentum probability densities for the Borromean exotic nuclei ${ }^{11} \mathrm{Li},{ }^{14} \mathrm{Be}$ and ${ }^{22} \mathrm{C}$. A fair reproduction of the experimental data was obtained in the case of the core recoil momentum distribution of ${ }^{11} \mathrm{Li}$ and ${ }^{14} \mathrm{Be}$ without resorting to free parameters.

We suggest a simple method to separate out from the cross-section data for the fragmentation of the ${ }^{22} \mathrm{C}$ the narrow peak in the core recoil momentum distribution associated with the breakup of the halo structure. For that aim, we performed the subtraction of a wide distribution associated with the core recoil momentum distribution observed for ${ }^{20} \mathrm{C}$. Indeed, the data for the fragmentation differential cross-section for the core momentum above $100 \mathrm{MeV} / \mathrm{c}$ in ${ }^{22} \mathrm{C}$ and ${ }^{20} \mathrm{C}$ seems indistinguishable, which indicates that the two neutrons in the wide part of the distribution come from inner part of the orbits localized within the core. The inverse of the difference between those cross-sections, after averaging them for positive and negative momentum, present a linear behaviour with $p^{2}$, which allows to extract the width of the momentum distribution. We apply the same fitting procedure to the difference of cross-sections but without the average. With these values at hand, we proceed to make a combined analysis of the width of the core recoil momentum distribution and matter radius for ${ }^{22} \mathrm{C}$.

We computed the scaling functions correlating s, $S_{2 n}$ and the virtual state energies of the two-body subsystems, and also the scaling functions correlating the matter radius with these quantities. The scaling function correlating s and $S_{2 n}$, with the experimental data for s equal to $31 \mathrm{MeV} / \mathrm{c}$ [11], $20 \pm 15 \mathrm{MeV} / \mathrm{c}$ and $34 \pm 21$ $\mathrm{MeV} / \mathrm{c}$, suggest that $S_{2 n}$ could be well above $100 \mathrm{keV}$ larger than previous estimations. From the correlation between the matter radius and $\mathrm{s}$, and the reference the value of $\mathrm{s}_{a v}=20 \pm 15 \mathrm{MeV} / \mathrm{c}$, one gets: $\sqrt{\left\langle r_{m}^{2}\right\rangle} \sim$ $3.5 \mathrm{fm}$ and $S_{2 n} \sim 400 \mathrm{keV}$. However, the values of 31 and $34 \pm 21 \mathrm{MeV} / \mathrm{c}$ (5) suggest much larger values of the two-neutron separation energy, about 800 and $900 \mathrm{keV}$, respectively. In these last two cases, the halo of ${ }^{22} \mathrm{C}$ would be quite small compared to the corresponding one in ${ }^{11} \mathrm{Li}$, and we believe that it would not justify the increase of the reaction cross section of ${ }^{22} \mathrm{C}$ compared to the neighbour nuclei, ${ }^{19} \mathrm{C}$ and ${ }^{20} \mathrm{C}$, as the experiment by Tanaka and collaborators [1] is showing. In addition, the clear evidence of the presence of a narrow peak in the core recoil momentum distribution, as pointed out by our model independent analysis, leads to $\mathrm{s}<20 \mathrm{MeV} / \mathrm{c}, S_{2 n}<400 \mathrm{keV}$ and $\sqrt{\left\langle r_{m}^{2}\right\rangle}>3.5 \mathrm{fm}$.

Our study also shows that the sensitivity of the width of the ${ }^{22} \mathrm{C}$ core momentum distribution to the virtual $s$-state energy of ${ }^{21} \mathrm{C}$ is quite small for $S_{2 n}$ fixed, but the matter radius is quite sensitive to it. The variation of the virtual state of ${ }^{21} \mathrm{C}$ from 0 to $1 \mathrm{MeV}$ shrinks considerably the matter radius. Such a tension between the 
halo size and the virtual state of ${ }^{21} \mathrm{C}$ suggests, within our analysis, that smaller values of $E_{n c}$ below $1 \mathrm{MeV}$ are preferred. Such conclusion is based on the belief that the Efimov physics, expressed by the calculated scaling laws, dominates the halo properties, and also by considering the validity of the Serber model to interpret the data on the core recoil momentum distribution. In short, the combined analysis of the core momentum distribution and matter radius for ${ }^{22} \mathrm{C}$, on the basis of the scaling laws computed close to the Efimov limit, suggest: (i) a virtual state of ${ }^{21} \mathrm{C}$ well below $1 \mathrm{MeV}$; (ii) the overestimation of the extracted matter ${ }^{22} \mathrm{C}$ radius; and (iii) a two-neutron separation energy between 100 and $400 \mathrm{keV}$.

Acknowledgments This work was partially supported by the Brazilian agencies CAPES, FAPESP and CNPq.

\section{References}

1. Tanaka, K., et al.: Observation of a large reaction cross section in the drip-line nucleus ${ }^{22}$ C. Phys. Rev. Lett. 104, 062701 (2010)

2. Yamashita, M.T., de Carvalho, R.M., Frederico, T., Tomio, L.: Constraints on two-neutron separation energy in the Borromean ${ }^{22}$ C nucleus. Phys. Lett. B 697, 90-93 (2011) [Addendum and erratum in Phys. Lett. B 715, 282-283 (2012)]

3. Fortune, H.T., Sherr, R.: Binding energy of ${ }^{22}$ C. Phys. Rev. C 85, 027303 (2012)

4. Ershov, S.N., Vaagen, J.S., Zhukov, M.V.: Binding energy constraint on matter radius and soft dipole excitations of ${ }^{22}$ C. Phys. Rev. C 86, 034331 (2012)

5. Acharya, B., Ji, C., Phillips, D.R.: Implications of a matter-radius measurement for the structure of Carbon-22. Phys. Lett. B 723, 196-200 (2013)

6. Acharya, B., Phillips, D.R.: Properties of Lithium-11 and Carbon-22 at leading order in halo effective field theory. arXiv:1508.02697 [nucl-th]

7. Wang, M., Audi, G., Wapstra, A.H., Kondev, F.G., MacCormick, M., Xu, X., Pfeiffer, B.: The Ame 2012 atomic mass evaluation. Chin. Phys. C 36, 1603-2014 (2012)

8. Gaudefroy, L., et al.: Direct mass measurements of ${ }^{19} \mathrm{~B},{ }^{22} \mathrm{C} 22,{ }^{29} \mathrm{~F},{ }^{31} \mathrm{Ne},{ }^{34} \mathrm{Na}$ and other light exotic nuclei. Phys. Rev. Lett. 109, 202503 (2012)

9. Mosby, S., et al.: Search for ${ }^{21} \mathrm{C}$ and constraints on ${ }^{22}$ C. Nucl. Phys. A 909, 69-78 (2013)

10. Ozawa, A., et al.: Measurements of interaction cross sections for light neutron-rich nuclei at relativistic energies and determination of effective matter radii. Nucl. Phys. A 691, 599-617 (2001)

11. Kobayashi, N., et al.: One- and two-neutron removal reactions from the most neutron-rich carbon isotopes. Phys. Rev. C 86, 054604 (2012)

12. Tanihata, I.: Neutron halo nuclei. J. Phys. G 22, 157-198 (1996)

13. Riisager, K.: Halos and related structures. Phys. Scr. T 152, 014001 (2013)

14. Souza, L.A., Bellotti, F.F., Yamashita, M.T., Frederico, T., Tomio, L.: Core momentum distribution in two-neutron halo nuclei. arXiv:1512.02070 [nucl-th]

15. Efimov, V.: Energy levels arising from resonant two-body forces in a three-body system. Phys. Lett. B 33, $563-564$ (1970)

16. Efimov, V.: Low-energy properties of three resonantly interacting particles. Sov. J. Nucl. Phys. 12 (1971), 589-595 [Yad. Fiz. 12, 1080-1091 (1970)]

17. Efimov, V.: Qualitative treatment of three-nucleon properties. Nucl. Phys. A 362, 45-70 (1981)

18. Yamashita, M.T., Bellotti, F.F., Frederico, T., Fedorov, D.V., Jensen, A.S., Zinner, N.T.: Single-particle momentum distributions of Efimov states in mixed-species systems. Phys. Rev. A 87, 062702 (2013)

19. Serber, R.: The production of high energy neutrons by stripping. Phys. Rev. 72, 1008-1016 (1947)

20. Hadizadeh, M.R., Yamashita, M.T., Tomio, L., Delfino, A., Frederico, T.: Effective range from tetramer-dissociation data for cesium atoms. Phys. Rev. A 87, 013620 (2013)

21. Gattobigio, M., Kievsky, A., Viviani, M.: Energy spectra of small bosonic clusters having a large two-body scattering length. Phys. Rev. A 86, 042513 (2012)

22. Kievsky, A., Gattobigio, M.: Universal nature and finite-range corrections in elastic atom-dimer scattering below the dimer breakup threshold. Phys. Rev. A 87, 052719 (2013)

23. Kievsky, A., Timofeyuk, N.K., Gattobigio, M.: N-boson spectrum from a discrete scale invariance. Phys. Rev. A 90, 032504 (2014)

24. Ji, C., Braaten, E., Phillips, D.R., Platter, L.: Universal relations for range corrections to Efimov features. Phys. Rev. A 92 , 030702(R) (2015)

25. Tanihata, I., Savajols, H., Kanungo, R.: Recent experimental progress in nuclear halo structure studies. Prog. Part. Nucl. Phys. 68, 215-313 (2013)

26. Smith, M., et al.: First penning-trap mass measurement of the exotic halo nucleus ${ }^{11}$ Li. Phys. Rev. Lett. 101, 202501 (2008)

27. Frederico, T., Tomio, L., Delfino, A., Hadizadeh, M.R., Yamashita, M.T.: Scales and universality in few-body systems. Few-Body Syst. 51, 87-112 (2011)

28. Frederico, T., Yamashita, M.T., Delfino, A., Tomio, L.: Universal aspects of light halo nuclei. Prog. Part. Nucl. Phys. 67, 939-994 (2012)

29. Zinner, N.T., Jensen, A.S.: Comparing and contrasting nuclei and cold atomic gases. J. Phys. G: Nucl. Part. Phys. 40, 053101 (2013)

30. Zahar, M., et al.: Momentum distributions for ${ }^{12,14}$ Be fragmentation. Phys. Rev. C 48, R1484-R1487 (1993)

31. Adhikari, S.K., Delfino, A., Frederico, T., Goldman, I.D., Tomio, L.: Efimov and Thomas effects and the model dependence of three-particle observables in two and three dimensions. Phys. Rev. A 37, 36663673 (1988) 
32. Skornyakov, G.V., Ter-Martirosyan, K.A.: Three body problem for short range forces. I. Scattering of low energy neutrons by deuterons. Sov. Phys. JETP 4, 648-661 (1957)

33. Phillips, A.C.: Consistency of the low-energy three-nucleon observables and the separable interaction model. Nucl. Phys. A 107, 209-216 (1968)

34. Yamashita, M.T., Frederico, T., Delfino, A., Tomio, L.: Scaling limit of virtual states of triatomic systems. Phys. Rev. A 66, 052702 (2002)

35. Yamashita, M.T., Tomio, L., Frederico, T.: Radii in weakly-bound light halo nuclei. Nucl. Phys. A 735, 40-54 (2004) 